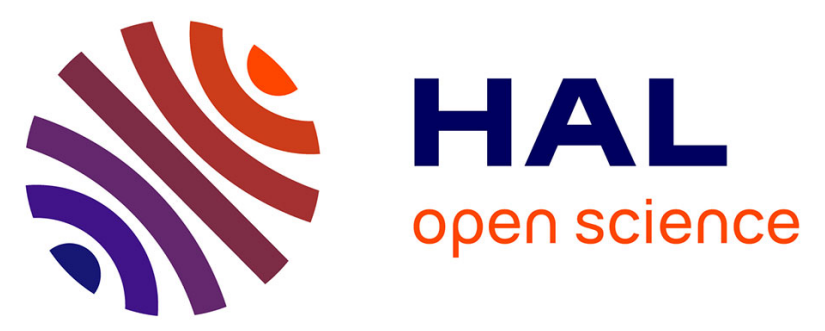

\title{
Single WDM channel efficient wavelength shifting and/or amplification using couterpropagating optical parametric amplifiers
}

Olivier Alibart, Marc de Micheli, Pascal Baldi

\section{To cite this version:}

Olivier Alibart, Marc de Micheli, Pascal Baldi. Single WDM channel efficient wavelength shifting and/or amplification using couterpropagating optical parametric amplifiers. Optics Communications, 2012, 285 (18), pp.3886-3889. 10.1016/j.optcom.2012.05.042 . hal-00849093

\section{HAL Id: hal-00849093 https://hal.science/hal-00849093}

Submitted on 30 Jul 2013

HAL is a multi-disciplinary open access archive for the deposit and dissemination of scientific research documents, whether they are published or not. The documents may come from teaching and research institutions in France or abroad, or from public or private research centers.
L'archive ouverte pluridisciplinaire HAL, est destinée au dépôt et à la diffusion de documents scientifiques de niveau recherche, publiés ou non, émanant des établissements d'enseignement et de recherche français ou étrangers, des laboratoires publics ou privés. 


\title{
Single WDM Channel Efficient Wavelength Shifting
}

\author{
and/or Amplification
}

\section{using Counterpropagating Optical Parametric Amplifiers}

\author{
Olivier Alibart, Marc De Micheli and Pascal Baldi* \\ Laboratoire de Physique de la Matière Condensée - UMR CNRS 6622, Université de Nice- \\ Sophia Antipolis, Parc Valrose, 06108 Nice Cedex 2, France
}

We theoretically and numerically study the difference frequency generation and the signal amplification bandwidth together with the pump wavelength acceptance for counterpropagating optical parametric amplifiers. It is shown that the specific advantage of those amplifiers is that the DFG and amplification bandwidth is much narrower than in a co-propagating scheme, thus making it possible to shift spectrally or to amplify selectively a single channel over the several present in WDM optical fiber communications. Moreover, the pump wavelength acceptance is shown to be as broad as in the copropagating scheme. The implementation of such counterpropagating optical parametric amplifiers in a quasi-phase-matched $\mathrm{LiNbO}_{3}$ waveguide is discussed.

Keywords: nonlinear optics; quasi-phase-matching; counterpropagating optical parametric interactions; integrated optics; optical communications; all-optical devices.

Corresponding author. Tel.: +33492076752; Fax: +33492076754 .

E-mail address: Pascal.Baldi@unice.fr. 


\section{Introduction}

The dramatic increase in recent years in the level of communication network traffic has initiated the development of all-optical communication devices and systems. The emergence of dense wavelength division multiplexing (WDM) requires both large bandwidth and single-channel frequency shifters and amplifiers. Co-propagating optical parametric amplifiers (OPA) based on quadratic nonlinear materials have been shown to be an interesting alternative to state-of-the-art semiconductor optical amplifiers and Raman and Er-doped fiber amplification systems at $1.55 \mu \mathrm{m}$ for large bandwidth amplification. Furthermore, wavelength conversion over a broad bandwidth comes naturally along with the OPA, which fulfill numerous requirements for ideal wavelength converters for telecommunications, such as high transparency, independence of bit rate and data format, and low cross talk. In this article we show that counterpropagating optical parametric amplifiers (COPA) offer the same advantages as OPA in terms of efficiency, tuning, tolerances... but can be used to efficiently shift spectrally and/or amplify only one single WDM channel.

The idea of COPA is not new [1], [2], but it has been subject to a revival of interest in the late 1990s thanks to the possibilities of implementation offered by the dramatic technological progress in the engineering of non-centrosymmetric crystals to achieve quasi-phase-matching (QPM) [3], particularly with respect to the reduction of achievable QPM grating periods. This technology, with high-order QPM gratings, has led to the first experimental demonstrations of contradirectional $\chi^{(2)}$ interactions in $\mathrm{LiNbO}_{3}$ [4] and KTP waveguides [5], namely backward second harmonic generation. More recently [6], a first order QPM counter-propagating optical parametric oscillator has been reported on bulk PPKTP but working far from degeneracy because of the too large QPM period $(800 \mathrm{~nm})$. Nevertheless, it is worth noting that experimental COPA 
has not been demonstrated so far. Furthermore, all the publications have been focused mainly on the amplification factor at perfect QPM, and COPAs have thus already been extensively studied for mirrorless optical parametric oscillation (OPO), efficient signal amplification [7] and cascading phase shift [8]. More precisely, the tolerances of the COPA to signal and pump wavelengths variations, which are key issues for practical applications, have not been discussed so far. Only a particular process, the spontaneous parametric down conversion, in a unguided pump configuration has been studied regarding the emitted fluorescence bandwidth [9], [10]. We show in this study that COPAs exhibit narrow wavelength conversion and signal amplification bandwidth and large pump wavelength acceptance. Both features would make the COPA suitable for efficiently spectrally shifting and/or amplifying single WDM channel, as opposed to the standard OPA scheme.

\section{COPA geometry and QPM curves}

The COPA has the traveling-wave geometry illustrated in Fig.1, with two inputs: a high-power pump wave at a wavelength $\lambda_{3}$, launched at $z=0$, and a counterpropagating low-power signal at $\lambda_{1}$ (entering at $z=L$ ). There are three outputs: the signal exiting at $z=0$ and the residual pump and the generated copropagating idler at $\lambda_{2}=\left(1 / \lambda_{3}-1 / \lambda_{1}\right)^{-1}$ that both exit at $z=L$. We emphasize that our definition of the signal and the idler waves is related to their directions of propagation and not to their wavelengths. $\lambda_{1}$ can thus be greater than $\lambda_{2}$, which is not the case with the traditional convention. It is worth noting that the configuration depicted in Fig. 1 is very convenient for amplification as the signal can easily be separated from the pump and the idler (by using an optical circulator for example). Nevertheless, such configuration can also be used for spectrally shifting as the idler can be separated from the pump because of their very different wavelengths (by using a WDM for example). We assume now that the nonlinear interaction 
occurs in a $\mathrm{LiNbO}_{3}$ waveguide with a generic $m$ th-order QPM. The period needed for $1^{\text {st }}$ order QPM is $\Lambda \sim 0.4 \mu \mathrm{m}$. For a typical soft-proton-exchanged (SPE) channel waveguide [11], where $N_{1}, N_{2}, N_{3}$ denote the mode indices at $\omega_{1}, \omega_{2}$ and $\omega_{3}$, respectively, $S_{\text {eff }}$ is the effective area (related to the modal overlap integral), and $d_{33}$ is the $\mathrm{LiNbO}_{3}$ quadratic tensor element, we define the interaction phase mismatch as $\Delta \beta=\left(N_{3} \omega_{3}+N_{1} \omega_{1}-N_{2} \omega_{2}\right) / c-2 \pi m / \Lambda\left[\mathrm{cm}^{-1}\right]$ and the coupling coefficient as $\kappa_{0}^{2}=\left(\mu_{0} / c\right)\left(\omega_{3}^{2} / 2\right)\left[2 d_{33} /(m \pi)\right]^{2}\left(S_{e f f} N_{1} N_{2} N_{3}\right)^{-1}\left[\mathrm{~W}^{-1} \mathrm{~cm}^{-2}\right]$. Finally, we express the proximity of the COPA to degeneracy by the factor $r_{1}=\left(2 \lambda_{3} / \lambda_{1}\right)$. To be efficient, the COPA must fulfill both energy and momentum conservation conditions:

$$
\begin{gathered}
\omega_{3}=\omega_{1}+\omega_{2} \\
\Delta \beta=\left(N_{3} \omega_{3}+N_{1} \omega_{1}-N_{2} \omega_{2}\right) / c-2 \pi m / \Lambda=0
\end{gathered}
$$

The corresponding QPM curves are presented in Fig. 2 for $m=1$ (first-order QPM) for different periods. Such curves are very different from standard QPM co-propagating curves ${ }^{10}$ as the counterpropagating signal has a nearly constant wavelength for a wide range of pump wavelength. We will see later on that this is responsible for the narrow signal amplification and spectral shifting bandwidths and the large pump wavelength acceptance of the COPA. In order to compute the difference frequency generation efficiency, the signal amplification factor and all the bandwidths, we now set $\lambda_{3} \sim 774 \mathrm{~nm}$ and $\lambda_{1} \sim 1.5 \mu \mathrm{m}$, which gives $\lambda_{2} \sim 1.6 \mu \mathrm{m}$. At those wavelengths, $r_{1}=1.03$ and the coupling coefficient is taken to be equal to the reported experimental value $\kappa_{0}^{2}=1.3 \mathrm{~W}^{-1} \mathrm{~cm}^{-2}$, which is very close to the calculated one $\left(\kappa_{0}^{2}=1.4 \mathrm{~W}^{-1} \mathrm{~cm}^{-2}\right)$ [11].

\section{Wavelength conversion efficiency and signal amplification factor}


In the stationary regime the $\chi^{(2)}$ interaction can be modeled through standard coupled-mode equations, written in terms of the slowly-varying envelopes $A_{\mathrm{i}}(z)$ at $\lambda_{\mathrm{i}}(i=1,2,3)$ :

$$
\left\{\begin{array}{l}
\frac{d A_{1}}{d z}=+i r_{1} \kappa_{0} A_{2}^{*} A_{3} \exp (-i \Delta \beta z) \\
\frac{d A_{2}}{d z}=-i\left(2-r_{1}\right) \kappa_{0} A_{1}^{*} A_{3} \exp (-i \Delta \beta z) \\
\frac{d A_{3}}{d z}=-i 2 \kappa_{0} A_{1} A_{2} \exp (+i \Delta \beta z)
\end{array}\right.
$$

where the amplitudes are normalized such that $\left|A_{i}(z)\right|^{2}=P_{i}(z)$ is the power carried by the mode at $\lambda_{i}$. The boundary conditions are then written as $\left|A_{3}(0)\right|^{2}=P_{30},\left|A_{2}(0)\right|^{2}=0$, and $\left|A_{1}(L)\right|^{2}=P_{1 L}$, with $P_{1 L}<<P_{30}$. To obtain the signal amplification factor $G_{1}=P_{1}(z=0) / P_{1 \mathrm{~L}}$ we employed a numerical technique to solve Eqs. (2) (a 'shooting' method with continuation) [12]. In Fig. 3, $G_{1}$ is plotted versus the normalized input pump power $\Gamma^{2}\left(=\kappa_{0}^{2} L^{2} P_{30}\right)$ for perfect phase matching $(\Delta \beta L=0)$ and for $P_{1 \mathrm{~L}}=6 \mathrm{~mW}$. On the uppermost horizontal axis we show the corresponding pump powers $\left(P_{30}\right)$ for first-order QPM in a SPE: $\mathrm{LiNbO}_{3}$ waveguide of length $L=3 \mathrm{~cm}$, with a normalized efficiency $\kappa_{0}^{2}=1.3 \mathrm{~W}^{-1} \mathrm{~cm}^{-2}$. Note that large amplification (more than $20 \mathrm{~dB}$ ) can be obtained at pump powers accessible to laser diodes. We have verified that the injected signal power $P_{1 \mathrm{~L}}$ does not affect $G_{1}$ as long as $P_{1 L}<<P_{30}$. In practice, this implies that $P_{1 \mathrm{~L}}$ should not exceed around 0.1 times $P_{30}$, which for significant values of $G_{1}$ corresponds to signal powers well above the powers used in WDM systems.

The difference frequency generation efficiency $G_{2}=P_{2}(z=L) / P_{1 \mathrm{~L}}$ is not represented in Fig. 3, as it is very close to $G_{1}$ except when $\Gamma^{2}$ tends to zero. In this case, $G_{2}$ tends to zero while $G_{1}$ tends to 1 (for a lossless medium). 
It is interesting to note that increasing $r_{1}$, which corresponds to move off degeneracy, increases the amplification factor, as long as $\kappa_{0}^{2}$ and thus $S_{\text {eff }}$ can be considered constant, but decreases the difference frequency generation efficiency. Nevertheless, $r_{1}$ must be close to 1 in order to keep the nonlinear interaction efficient and to consider $S_{\text {eff }}$ constant, so the effect of such small variations of $r_{1}$ is negligible.

\section{Signal and idler bandwidths and pump wavelength acceptance}

For a given $\Gamma^{2}$, we then plotted $G_{1}$ as a function of $\Delta \beta L$ and deduced from that curve its full width at half maximum (FWHM) $\delta$. This FWHM is presented in Fig. 4 as a function of $\Gamma^{2}$ and will be used in the following to calculate the signal amplification bandwidth $\Delta \omega_{1}$ and the pump wavelength acceptance $\Delta \omega_{3}$. Because of the energy conservation condition expressed in Eq. (1a), the idler (or spectral shifting) bandwidth $\Delta \omega_{2}$ is equal to $\Delta \omega_{1}$.

Keeping $\lambda_{3}$ constant, we calculated $\Delta \omega_{1}$ corresponding to $\delta$ by expanding $\Delta \beta$ around the perfect QPM condition given by Eq. (1b) and by using the derivative of Eq. (1a) [13]:

$$
\Delta \omega_{1} L=\delta c /\left|N_{1}+N_{2}+\left(\partial N_{1} / \partial \omega\right) \omega_{1}+\left(\partial N_{2} / \partial \omega\right) \omega_{2}\right|=\left(2 \pi c / \lambda_{1}^{2}\right) \Delta \lambda_{1} L
$$

For example, if we consider $G_{1}=10$, obtained for $\Gamma^{2}=2.03$, Fig. 4 indicates that $\delta=1.6$, which gives $\Delta \lambda_{1} L=0.02 \mathrm{~nm} . \mathrm{cm}$ for the wavelengths that we consider. This is 3 orders of magnitude lower than for the standard copropagating OPA. Let us now call $\delta v_{1}$ the spacing between two adjacent WDM channels and $d v_{1}$ the bandwidth of each channel. In order to amplify one single

channel $d v_{1} L \leq \frac{c}{\lambda_{1}^{2}} \Delta \lambda_{1} L \leq \delta v_{1} L$. If we first consider a U-WDM grid spacing of $10 \mathrm{GHz}$, this means that for achieving $G_{1}=10$ the waveguide length must be longer than $0.3 \mathrm{~cm}$ but not more 
than $3 \mathrm{~cm}$ if we want to allow $1 \mathrm{Gbit} / \mathrm{s}$ per channel. According to Fig. 3, this implies that $P_{30} \approx 180 \mathrm{~mW}$ in the case of $L=3 \mathrm{~cm}$. If we now consider a WDM spacing of $100 \mathrm{GHz}$ and a bandwidth of $10 \mathrm{GHz}$, then $0.03 \mathrm{~cm} \leq L \leq 0.3 \mathrm{~cm}$, which implies that $P_{30} \approx 18 \mathrm{~W}$ for $G_{1}=10$. On the one hand those values indicate that centimeter long COPA is very attractive for efficiently amplifying or spectrally shifting a single WDM channel. On the other hand this clearly shows that the waveguide length, and therefore the parametric gain for a given pump power, is limited by the bit rate to be amplified.

Following the same approach, we expressed the pump wavelength acceptance $\Delta \omega_{3}$ for a given $\lambda_{1}$ :

$$
\Delta \omega_{3} L=\delta c /\left|N_{3}-N_{2}+\left(\partial N_{3} / \partial \omega\right) \omega_{3}-\left(\partial N_{2} / \partial \omega\right) \omega_{2}\right|=\left(2 \pi c / \lambda_{3}^{2}\right) \Delta \lambda_{3} L
$$

For example, if we consider $G_{1}=10(\delta=1.6), \Delta \lambda_{3} L=0.7 \mathrm{~nm}$.cm for the wavelengths that we consider. This expression is the same as for the standard copropagating OPA, which show that COPA has a large pump wavelength acceptance.

It is very important to note that changing $r_{1}$, i.e. the signal and idler wavelengths, does not affect significantly $\Delta \lambda_{1} L$, apart from the dispersion of the mode indices $N_{1}$ and $N_{2}$. This indicates that the signal amplification bandwidth remains almost constant over a wide range of wavelengths. Such situation is completely different from the co-propagating configuration, for which $\Delta \lambda_{1} L$ dramatically increases when $r_{1}$ tends to 1 [14]. Furthermore, we have verified that the propagation losses do not change $\Delta \lambda_{1} L$.

Both narrow signal amplification bandwidth and large pump wavelength acceptance can be understood by looking at Fig. 2, where we see that for a wide range of pump wavelength, the counterpropagating signal has a nearly constant wavelength. We can also consider the 
wavevector diagram depicted in Fig. 5. If we keep $\lambda_{3}$ constant, the conservation of energy implies that increasing $\lambda_{1}$ will decrease $\lambda_{2}$. But increasing (respectively decreasing) $\lambda$ means decreasing (respectively increasing) the corresponding wavevector. Thus the effect of decreasing the wavevector at $\lambda_{1}$ will be enhanced by the increasing of the wavevector at $\lambda_{2}$, leading to a large phase-mismatch $\Delta \beta$ for small variation of $\lambda_{1}$. Conversely, if we keep $\lambda_{1}$ constant, the conservation of energy implies that increasing $\lambda_{3}$ will increase $\lambda_{2}$, so the two similar variations of the wavevectors at $\lambda_{3}$ and $\lambda_{2}$ will compensate for each other, leading to a small phase-mismatch $\Delta \beta$ for large variation of $\lambda_{3}$.

It is worth noting that permutating signal and idler, i.e. having the signal co-propagating with the pump, would on the one hand not change the gain and the signal and idler bandwidths of the COPA. This is simply due to the symmetry that exists between the signal and the idler waves, symmetry that appears in Eq. (3). On the other hand, such permutation would dramatically change the pump wavelength acceptance. Indeed, the two “_“ signs in Eq. (4) should be replaced by two "+" signs, which in turns would decrease $\Delta \omega_{3} L$ to approximately $\Delta \omega_{1} L$.

\section{Discussion}

The power requirements of the proposed device depend strongly on the ability to achieve loworder QPM over centimeter lengths. We can estimate the nonlinear coupling coefficient of the COPA with $m^{\text {th }}$ order QPM as $\kappa_{0}^{2} \sim \eta_{\text {nor }} / m^{2}$, where $\eta_{\text {nor }}$ is the normalized efficiency for the equivalent copropagating OPA at similar wavelengths in SPE: $\mathrm{LiNbO}_{3}$ waveguides with $1^{\text {st }}$ order QPM $\left(\eta_{\text {nor }}=130 \% \mathrm{~W}^{-1} \mathrm{~cm}^{-2}\right)[11]$. On the upper horizontal axis of Fig. 3 we converted the values of $\Gamma^{2}$ into real powers $\left(P_{30}\right)$ for a 3-cm-long waveguide and $m=1$. For higher-order QPM the figures have to be scaled by $m^{-2}$. For instance, point $\Gamma^{2}=2.03$ in Fig. 3 would correspond to 
$P_{30} \approx 180 \mathrm{~mW}$ for $m=1(\Lambda=0.4 \mu \mathrm{m})$ and to $P_{30} \approx 8.8 \mathrm{~W}$ for $m=7$, i.e., with $\Lambda=2.8 \mu \mathrm{m}$, well within state-of-the-art poling capabilities [4]. Note that the required power is inversely proportional to the square of the device length.

Even if the technology of SPE [10] (or APE/RPE [15] and Ti:indiffusion [16]) is now mature and allow the fabrication of uniform waveguides over 3 inches wafers, it is interesting to estimate the impact of waveguide non-uniformity on the efficiency and on the signal bandwidth of the proposed device. Waveguide non-uniformity could come for example from a temperature gradient in heated samples or from QPM pattern errors. For the co-propagating configuration, the influence of such non-uniformity has been studied in details by Fejer et al. [17]. Because of the direct analogy between Eqs. (2), (1b) and (3) respectively and Eq. (1), (20) and (24) of reference 16 respectively, the results reported in [17] can be directly used in our case. For example, let us consider a linear taper of period with a slope of $0.1 \%$. Eq. (84) of reference 16 indicates that, for a 3-cm-long waveguide, the parameter $\xi$ is equal to 4 for $m=1$ and to 1 for $m=7$. In the first case, the amplification efficiency would be reduced by a factor of 15 compared to a uniform waveguide while the amplification bandwidth would be increased by a factor of 15 . In the second case, the efficiency would be reduced by $25 \%$ while the bandwidth would be increased by a factor of 2 .

This study has been performed using a quasi-CW approach, which is coherent with the bit rate limitation. Indeed, $d v_{1} L \leq \frac{\Delta \lambda_{1} L}{\lambda_{1}^{2}} c$ (see section IV), so $d v_{1} L \leq 0.1 c$ for telecom wavelengths. This means that $d v_{1} L \leq v_{g}\left(\lambda_{1}\right)$, where $v_{g}\left(\lambda_{1}\right)$ is the group velocity at $\lambda_{1}$, which corresponds to the quasi-CW condition. It is also clear that, as any other quadratic nonlinear process, COPA is polarization sensitive. If both wave polarizations and crystal orientation are 
not adequate, COPA does not occur and the travelling wave do not interact, unlike in a Kerr medium [18].

\section{Conclusion}

For the first time to the best of our knowledge, we have investigated the response of a COPA structure in terms of its tolerances to signal and pump wavelengths variations. Both narrow signal amplification and wavelength shifting bandwidths and large pump wavelength acceptance make the COPA appealing for all-optical amplifying and/or spectrally shifting a single WDM channel. Whereas peak powers of the order of $10 \mathrm{~W}$ are required for demonstration of these effects in with the present-technology $\mathrm{SPE}: \mathrm{LiNbO}_{3}$ waveguides, sub-watt excitation can be expected for first-order QPM when the domain backswitch technique [19] or the overpoling technique [20] are used.

We thank D.B. Ostrowsky and J.-S. Tanzilli for stimulating discussions. This research was sponsored by the CNRS and the Région PACA through the co-financed grant 01/11159. 
Figures and figure captions

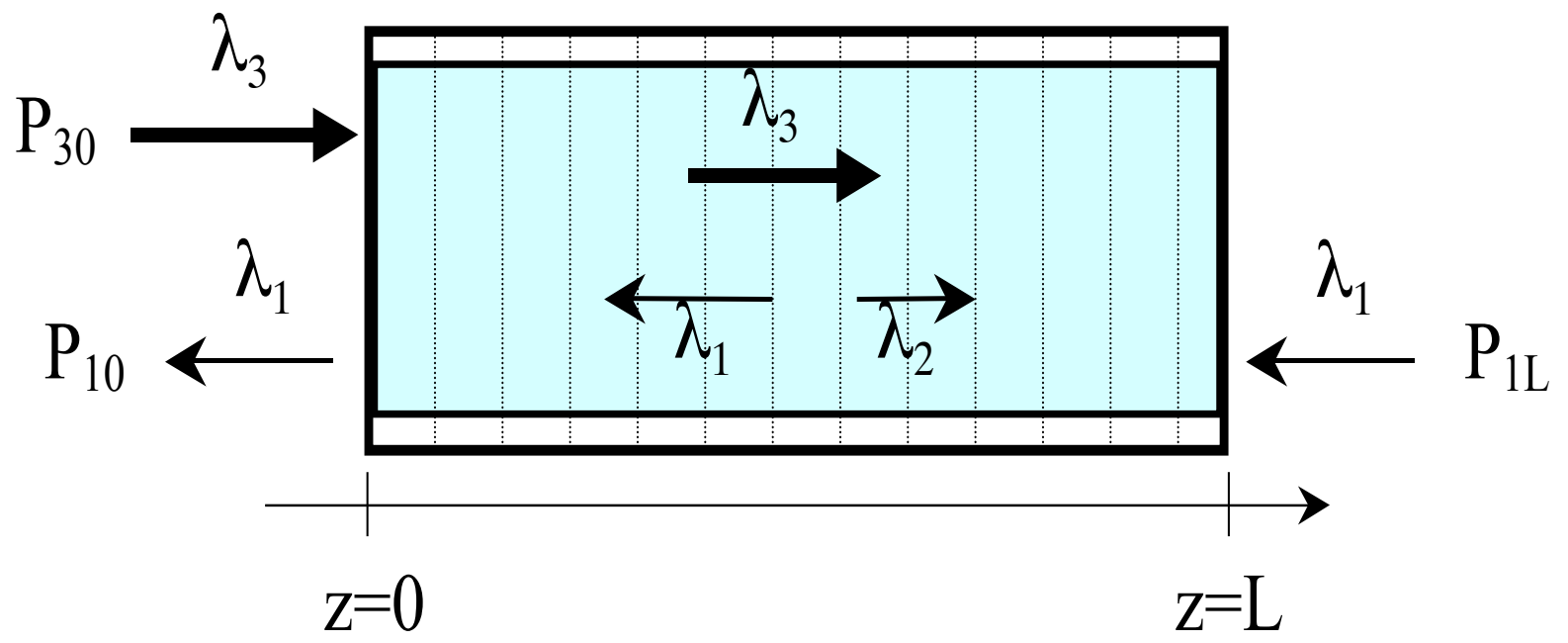

1. Scheme of the COPA 


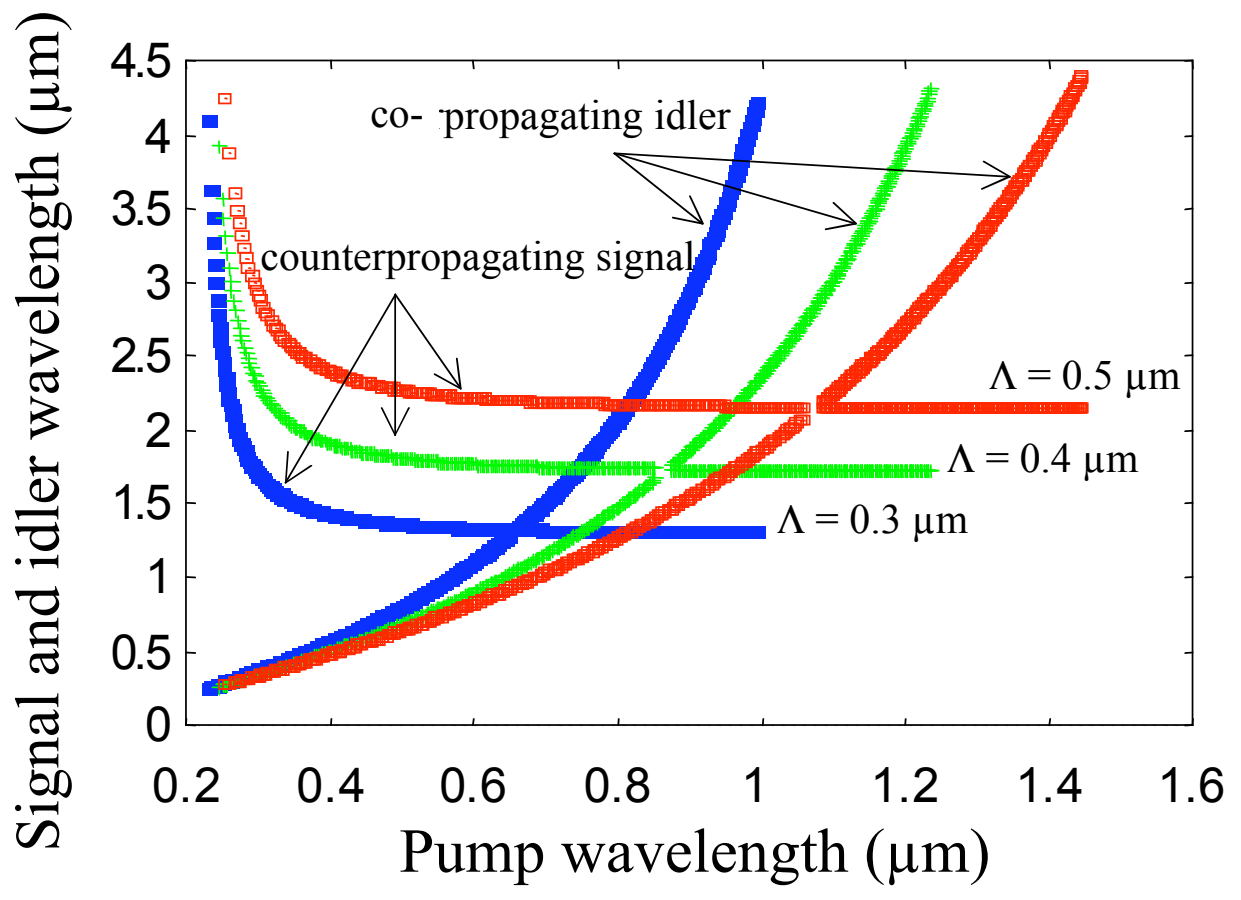

2. First-order QPM curves of the COPA. Please note that we do not follow the convention $\lambda_{\text {idler }}>\lambda_{\text {signal }}$ (see text for details). 


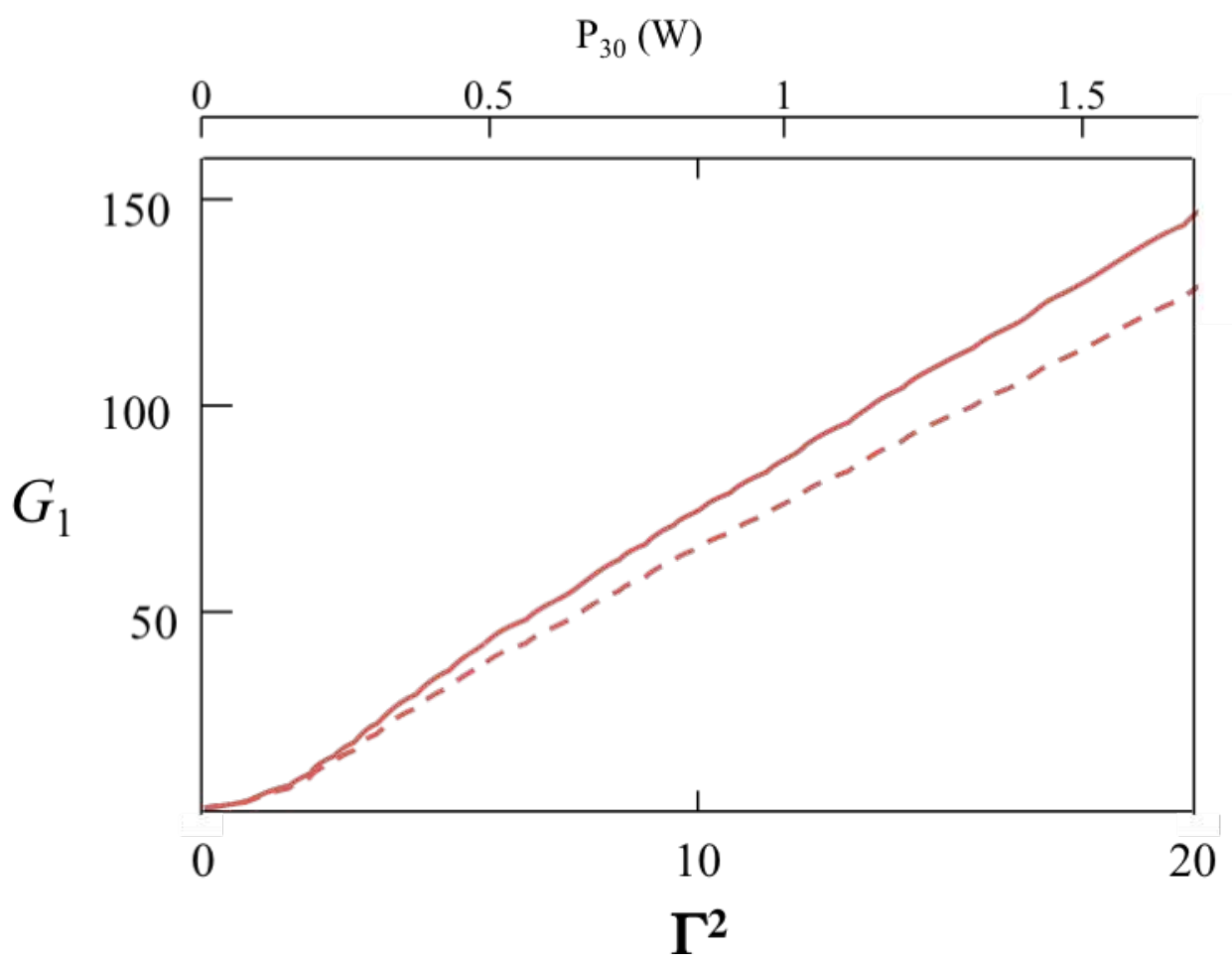

3. Signal amplification $\left[G_{1}=P_{1}(z=0) / P_{1 \mathrm{~L}}\right]$ versus normalized input pump power $\left(\Gamma^{2}=\kappa_{0}^{2} L^{2} P_{30}\right)$, for perfect quasi-phase-matching $\Delta \beta L=0$ with $r_{1}=1.03$ and $\kappa_{0}^{2} L^{2} P_{1 \mathrm{~L}}=0.07$. On the uppermost horizontal axis we show the corresponding pump powers $\left(P_{30}\right)$ for first-order QPM in a SPE: $\mathrm{LiNbO}_{3}$ waveguide of length $L=3 \mathrm{~cm}$, with a normalized efficiency $\kappa_{0}^{2}=1.3 \mathrm{~W}^{-1} \mathrm{~cm}^{-2}$. The corresponding injected signal power is in this case $P_{1 \mathrm{~L}}=6 \mathrm{~mW}$. Solid curve: $G_{1}$ for a lossless medium; dashed curve: $G_{1}$ for typical SPE waveguide losses $\left(\alpha_{1}=\alpha_{2}=0.2 \mathrm{~dB} / \mathrm{cm}\right.$ and $\left.\alpha_{3}=0.4 \mathrm{~dB} / \mathrm{cm}\right)$. 


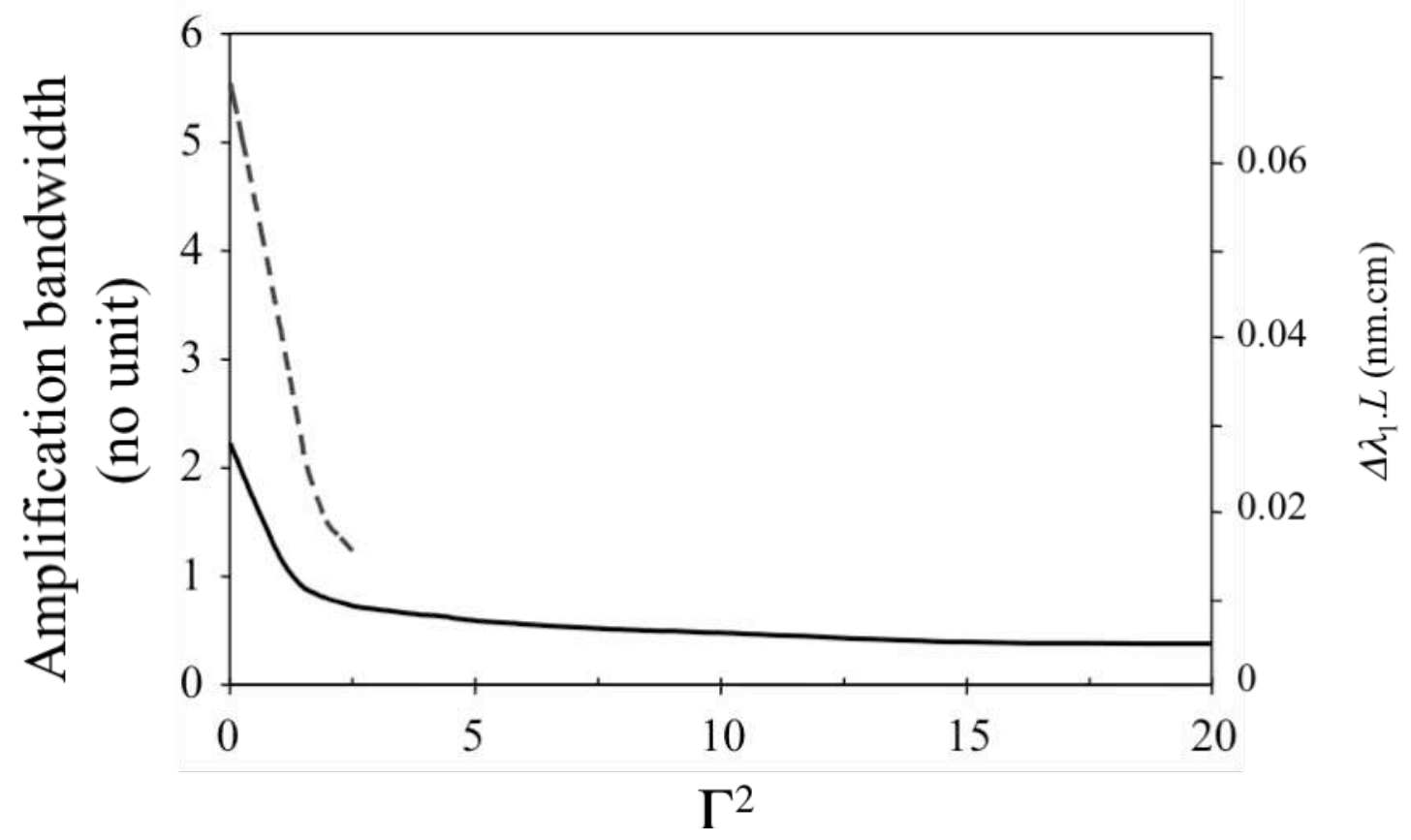

4. Signal amplification bandwidth versus normalized input pump power $\left(\Gamma^{2}=\kappa_{0}^{2} L^{2} P_{30}\right)$, with $r_{1}=1.03$ and $\kappa_{0}^{2} L^{2} P_{1 \mathrm{~L}}=0.07$. The dashed curve represents the FWHM $\delta$ while the solid curve represents the width at $90 \%$ of the maximum. $\delta$ no longer exists for values of $\Gamma^{2}$ above 2.47 because of the multivalued response of the COPA. ${ }^{4}$ The right vertical axis shows the corresponding product $\Delta \lambda_{1} L$ for a SPE: $\mathrm{LiNbO}_{3}$ waveguide with $\lambda_{1}=1.5 \mu \mathrm{m}$. 


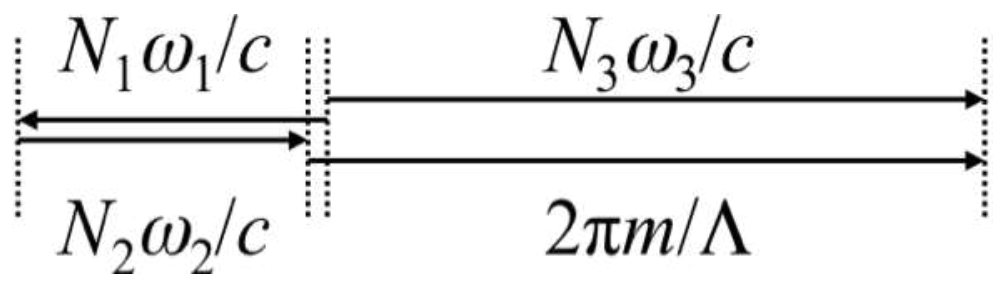

5. Wavevectors diagram of the COPA. 


\section{References}

[1] N. M. Kroll, " Parametric amplification in spatially extended media and application to the design of tuneable oscillators at optical frequencies ", Phys. Rev. 127 (1962) 1207-1211.

[2] S. E. Harris, " Proposed backward wave oscillation in the infrared ", Appl. Phys. Lett. 9 (1966) 114-116.

[3] J. A. Armstrong, N. Bloembergen, J. Ducuing and P. S. Pershan, " Interactions between light waves in a nonlinear dielectric ", Phys. Rev. Lett. 127 (1962) 1918-1939.

[4] X. Gu, R. Korotkov, Y. J. Ding, J. U. Kang, J. B. Khurgin, " Backward second-harmonic generation in periodically poled lithium niobate ", J. Opt. Soc. Am. B 15 (1998) 1561-1566.

[5] X. Gu, M. Makarov, and Y. J. Ding, J. B. Khurgin, W. P. Risk, " Backward second-harmonic and third-harmonic generation in a periodically poled potassium titanyl phosphate waveguide ", Opt. Lett. 24 (1999) 127-129.

[6] C. Canalias and V. Pasiskevicius, "Mirrorless optical parametric oscillator ", Nature Photonics 1 (2007) 459.

[7] Y. J. Ding and J. B. Khurgin, " Backward optical parametric oscillators and amplifiers ", IEEE J. Quantum Electron. 32 (1996) 1574-1582.

[8] K. Gallo, P. Baldi, M. De Micheli, and D.B. Ostrowsky; G. Assanto, " Cascading phase shift and multivalued response in counterpropagating frequency-nondegenerate parametric amplifiers ", Opt. Lett. 25 (2000) 966-968.

[9] A. De Rossi and V. Berger, " Counterpropagating twin photons by parametric fluorescence ", Phys. Rev. Lett. 88 (2002) 043901. 
[10] M. C. Booth, M. Atatüre, G. Di Giuseppe, B. E. A. Saleh, A. V. Sergienko and M. C. Teich, " Counterpropagating entangled photons from a waveguide with periodic nonlinearity ", Phys. Rev. A 66 (2002) 023815.

[11] L. Chanvillard, P. Aschiéri, P. Baldi, D. B. Ostrowsky, and M. P. De Micheli; L. Huang and D. J. Bamford, " Soft Proton Exchange on PPLN: a simple waveguide fabrication process for highly efficient nonlinear interactions ", Appl. Phys. Lett. 76 (2000) 1089-1091.

[12] Seydel, Practical Bifurcation and stability analysis, Springer ed., New York (1994).

[13] R.L. Byer, in Quantum Electronics, H. Rabin and C.L. Tang eds., Academic Press, New York (1977).

[14] P. Baldi, M.L. Sundheimer, K. El Hadi, M. de Micheli and D.B. Ostrowsky, " Comparison between difference-frequency generation and parametric fluorescence in quasi-phase-matched lithium niobate stripe waveguides ", Sel. Topics in Quantum Electronics (Integrated Optics) 2 (1996) 385-395.

[15] K. R. Parameswaran, R. K. Route, J. R. Kurz, R. V. Roussev, and M. M. Fejer ; M. Fujimura, "Highly efficient second-harmonic generation in buried waveguides formed by annealed and reverse proton exchange in periodically poled lithium niobate ", Opt. Lett. 27 (2002) 179-181.

[16] G. Schreiber, H. Suche, Y. L. Lee, W. Grundkötter, V. Quiring, R. Ricken and W. Sohler, "Efficient cascaded difference frequency conversion in periodically poled $\mathrm{Ti}: \mathrm{LiNbO}_{3}$ waveguides using pulsed and cw pumping ", Appl. Phys. B 73 (2001) 501-504.

[17] M.M. Fejer, G.A. Magel, D.H. Jundt and R.L.Byer, "Quasi-phase-matched second harmonic generation: tuning and tolerances ", IEEE J. Quant. Elec. 28 (1992) 2631-2654. 
[18] S. Pitois, G. Millot and S. Wabnitz, "Nonlinear polarization dynamics of counterpropagating waves in an isotropic optical fiber: theory and experiments ", JOSA B 18 (2001) 432-443.

[19] V. Ya Shur, E. L. Rumyantsev, E. V. Nikolaeva, E. I. Shishkin, D.V. Fursov, R.G. Batchko, L.A. Eyres, M.M. Fejer and R.L. Byer, " Nanoscale backswitched domain patterning in lithium niobate ", Appl. Phys. Lett. 76 (2000) 143-145.

[20] A. C. Busacca, C. L. Sones, V. Apostolopoulos, R. W. Eason, and S. Mailis, " Surface domain engineering in congruent lithium niobate single crystals: A route to submicron periodic poling ", Appl. Phys. Lett. 81 (2002) 4946-4948. 